\title{
Influence of Internal Discharges in a Cavity within Polymeric Insulating Material Caused by Partial Discharge Mechanisms
}

\author{
T. Tanmaneeprasert*, P.L. Lewin, and J.A. Hunter \\ School of Electronics and Computer Science \\ University of Southampton \\ Southampton, United Kingdom \\ *E-mail: tt1e14@ecs.soton.ac.uk
}

\begin{abstract}
This paper presents the time varying characteristics of partial discharge (PD) activity in a cylindrical cavity within polymeric insulation. The samples are produced using a multiple layer technique with polyethylene terephthalate (PET) selected as the solid dielectric. The results highlight the degradation process of the cavity surface through the exposure to a $\mathrm{HV}$ AC electric field and the impact of this on PD activity. The variation in sample conditions are linked to PD analysis in each case with one sample having exhibited electrical tree growth. The morphological surface and chemical composites of the test samples are analysed using Raman spectroscopy and an optical microscope.
\end{abstract}

Keywords-partial discharge; cylindrical cavity; polyethylene terephthalate; degradation process; electrical tree growth; Raman spectroscopy.

\section{INTRODUCTION}

Partial discharge diagnostics have been applied as an effective condition monitoring tool for assessing dielectric degradation processes for more than a half century [1]. The analysis of phase resolved partial discharge (PRPD) patterns has been investigated for source classification purposes in insulating electric cables [2]. One of the recognized degradation techniques involved the generation of internal discharges such that the transition of electric tree growth leads to full insulation failure. Previous work in this area was identified the PD characteristics of the typical cavity discharge activity in terms of turtle-like or rabbit-like patterns [3-5]. However, electrical tree growth was linked to the transition of wing-like patterns $[6,7]$ associated with the appearance of swarming pulsive microdischarge (SPMD) mechanisms [8-10]. In this paper, a cylindrical cavity was embedded in the middle layer of a three layer polyethylene terephthalate (PET) sample. The characteristics of PRPD patterns were investigated during electrical ageing over a period of 38 hours. The variation in morphology and the formation of chemical composites were determined using both Raman spectroscopy and optical imaging, where evidence of electrical tree growth was identified.

\section{EXPERIMENTAL SETUP}

\section{A. Experimental arrangement of the test object}

The samples were made using three layers of Polyethylene terephthalate (PET) films with a thickness of $0.3 \mathrm{~mm}$ and a cylindrical cavity of diameter $1.1 \mathrm{~mm}$ in the center of the middle layer. The samples were placed between two parallel brass plane electrodes with a diameter and thickness of $10 \mathrm{~mm}$ and $20 \mathrm{~mm}$ respectively. The upper electrode was connected to a variable $20 \mathrm{kV} \mathrm{HV} \mathrm{AC} \mathrm{supply} \mathrm{and} \mathrm{the} \mathrm{lower} \mathrm{electrode} \mathrm{was} \mathrm{connected} \mathrm{to}$ ground as shown in Figure 1.

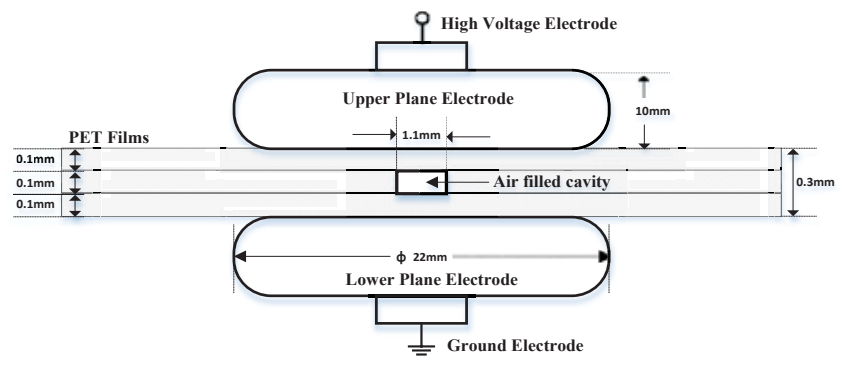

Figure 1. Electrode arrangement of sample with a cavity.

\section{B. Partial discharge measurement}

PD measurement within the experiment was achieved using an Omicron Mtronix MPD 600 system, which generated phase resolved partial discharge (PRPD) patterns throughout the deterioration process. The PRPD measurement system was calibrated using an Omicron Mtronix CAL 542 unit before the application of HV. The applied voltage level for testing was calculated by finding the average partial discharge inception voltage (PDIV) for 11 test samples (approximately $2 \mathrm{kV}$ ) and multiplying each sample by a factor of 1.5 times $(3 \mathrm{kV})$. All HV connections were made using copper pipes in order to suppress corona. The sample and electrode arrangement was immersed in silicone oil to prevent surface discharges with a schematic of the test arrangement as shown in Figure 2.

\section{RESULTS OF PRPD PATTERNS DURING THE AGEING PROCESS}

During the electrical ageing process, visual monitoring of the PRPD patterns were completed at 30 minute intervals in the event that successive patterns differed, the new plot was recorded and evidence of sample ageing recorded. This relied on the assumption that if no deterioration of the sample had occurred, the response of the defect to the applied voltage (phase resolved histogram) would remain consistent. 
For the purposes of this experiment, two of the eleven samples were randomly selected for PD measurements during the ageing process.

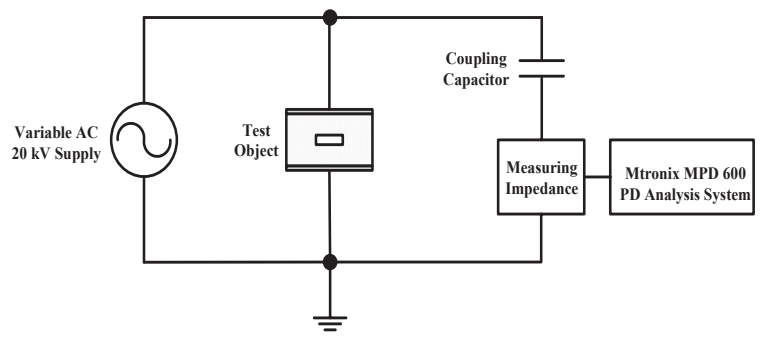

Figure 2. Partial discharge measuring system.

\section{A. Characteristics of rabbit-like PRPD patterns}

The first sample (sample one) exhibited a clear transition of PRPD patterns during the ageing process. After a period of one hour, the PRPD pattern was identified as a rabbit-like shape, where the charge magnitude exhibited symmetry between the positive and negative half cycles as shown in Figure 3 (a). This pattern of activity remained consistent until the transition to growth in the rabbit ear was observed after 16 hours as shown in Figure 3 (b).

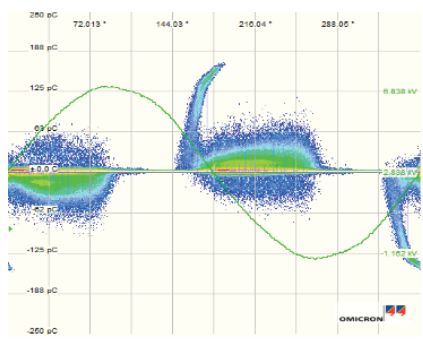

(a)

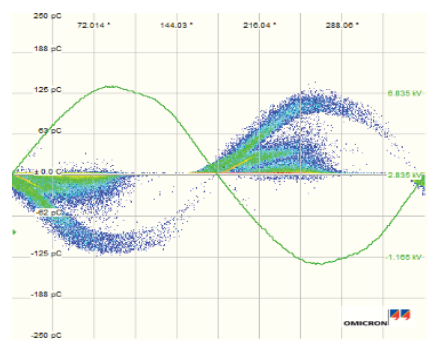

(b)
Figure 3. The variation in rabbit-like patterns in the sample one with ageing time at $3 \mathrm{kV}$; (a) at 1 hour and (b) at 16 hours.

\section{B. Characteristics of wing-like PRPD patterns}

A typical wing-like pattern was detected after 20 hours as shown in Figure 4 (a). At 22 hours into the test, the average pulse magnitude was recorded as less than $1 \mathrm{pC}$. The average number of PDs per cycle had also dropped by $90 \%$, with the pattern shown in Figure 4 (b). At this stage, an investigation into the morphology of the test sample was undertaken with evidence of electrical tree growth on the cavity wall as further detailed in Section IV.

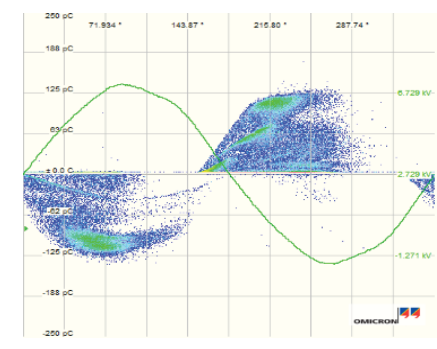

(a)

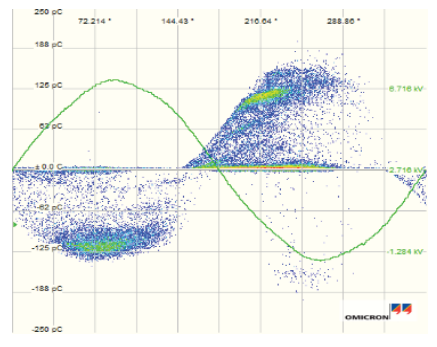

(b)
Figure 4. The variation in wing-like patterns in the sample one with ageing time at $3 \mathrm{kV}$; (a) at 20 hours and (b) at 22 hours after occurring the SPMD.

\section{Influence of PRPD patterns after the SPMD mechanism}

After the identification of SPMD activity had occurred, the experiment was restarted under the same conditions. The rabbit-like PRPD pattern was recorded after a total test time of 23 hours. After a further 25 hours, the space between the part of rabbit ear and body in this pattern were interlinked as shown in Figure 5 (a) and (b) respectively.

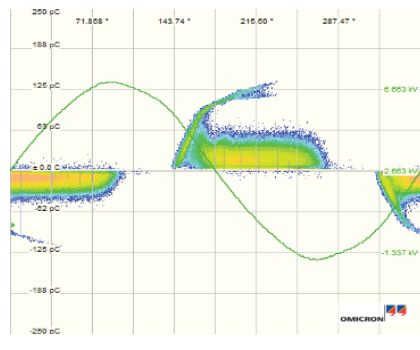

(a)

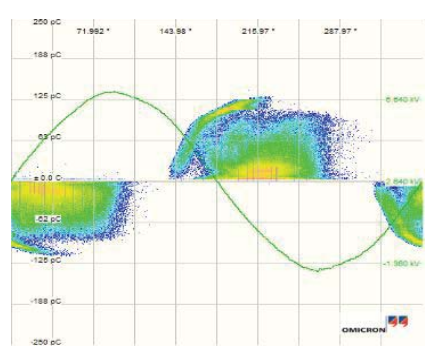

(b)
Figure 5. The variation in rabbit-like patterns in the sample one upon reenergisation had occurred the SPMD at $3 \mathrm{kV}$; (a) at 23 hours and (b) at 25 hours.

\section{Characteristics of turtle-like PRPD patterns}

A typical turtle-like pattern was noticed after a period of 30 hours as shown in Figure 6 (a). After 38 hours, the phase relationship in the PRPD pattern was noticed to expand with PD occurring across the entire power cycle as shown in Figure 6 (b).

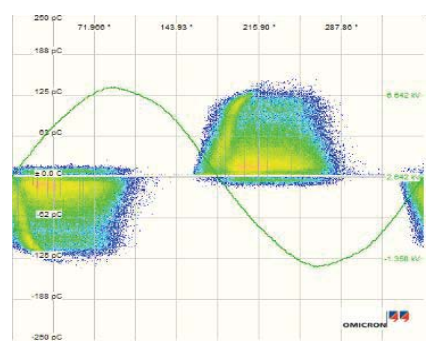

(a)

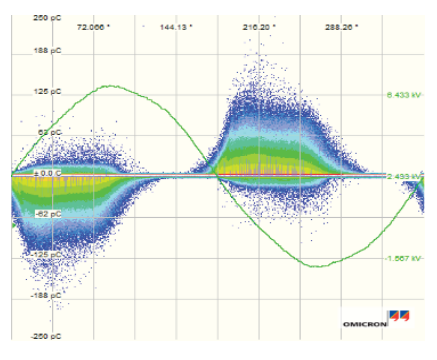

(b)
Figure 6. The variation in turtle-like patterns in the sample one with ageing time at $3 \mathrm{kV}$; (a) at 30 hours and (b) at 38 hours.

\section{E. Characteristic sequences of the PRPD analysis}

The PRPD characteristics during the degradation process were divided into two stages of the SPMD consequence as shown in Figure 7.

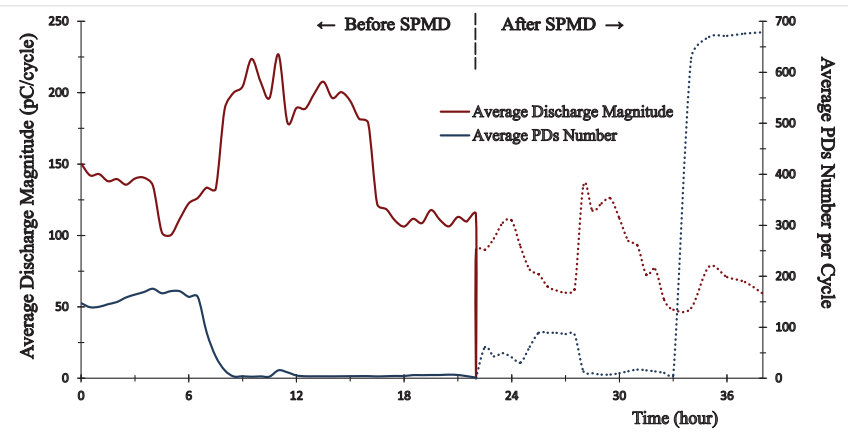

Figure 7. The behaviour sequences of the sample one with ageing time; average discharge magnitude and PD number as red and blue lines respectively. 
In the period before the SPMD, the PRPD patterns changed from the rabbit-like into wing-like until the PD extinction event at 22 hours. At this stage, an investigation into the morphology of the sample was undertaken with evidence of deterioration of the cavity surface and a burst cavity are further detailed in Section IV.

\section{$F$. Influence of PD characteristics in other samples}

In the case of the second sample (sample two), the PRPD data was linked to the rabbit-like shape after 1 hour. While the notable variation in the PRPD pattern was a larger ear detected over a time period of 6 hours as shown in Figure 8 (a) and (b) respectively. The significant consequence of PRPD patterns was changed into the turtle-like pattern after 16 hours. This transition was acquired to continuous processes until a time period of 35 hours as shown in Figure 8 (c) and (d) respectively.

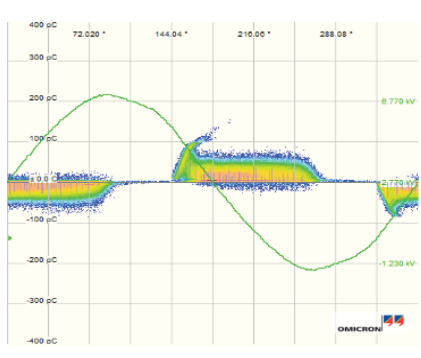

(a)

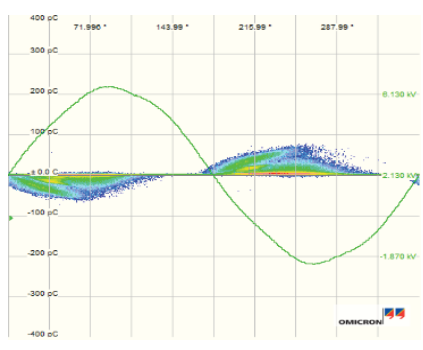

(c)

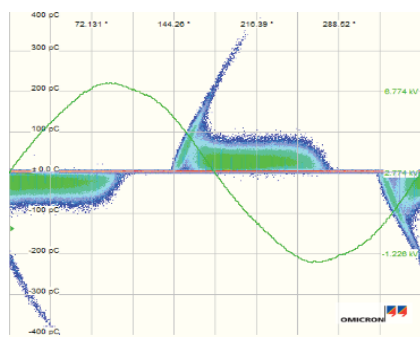

(b)

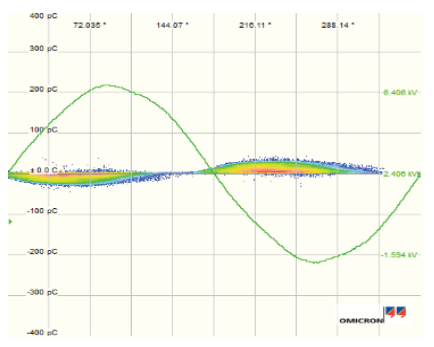

(d)
Figure 8. The variation in PD patterns in the sample two with ageing time at $3 \mathrm{kV}$; (a) at 1 hour, (b) at 6 hours, (c) at 16 hours and (d) at 35 hours.

\section{MORPHOLOGY AND RAMAN ANALYSIS RESULTS}

The significant regions on the cavity surface of sample one were analysed using both Raman spectroscopy and a standard microscope at several stages of the ageing process.

\section{A. Morphology of the tree growth on the cavity surface}

Throughout the ageing experiment, the morphology in various points were revealed to deteriorate on the cavity surface and the electrical tree growth were observed. Referring to Figures 9 and 10, the surface roughness areas are indicated with arrows marked as (A) and the inner cavity (B) after ageing time at 33 hours, these regions were observed with increasing roughness on the cavity surface and the inner part of the cavity with a dark surface area after ageing time at 38 hours as shown in Figure 9 (a) and (b) respectively. The electrical tree growth where the red arrow was marked as (A), which was noticed at the time period of 22 hours and 33 hours respectively as shown in Figure 10 (a) and (b). Significantly, the thickness of the electrical tree and additional branches increased after ageing for 33 hours as shown in Figure 10 (b).

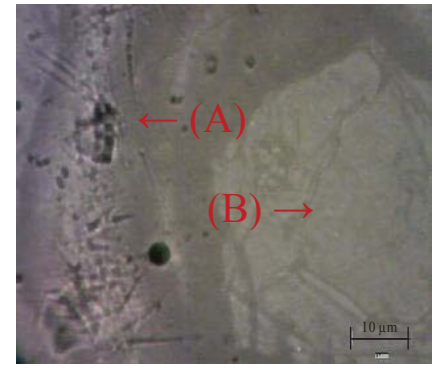

(a)

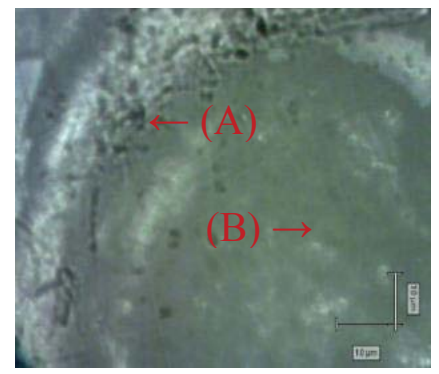

(b)
Figure 9. Morphology on the cavity surface in the sample one after ageing time; (a) at 33 hours and (b) at 38 hours.

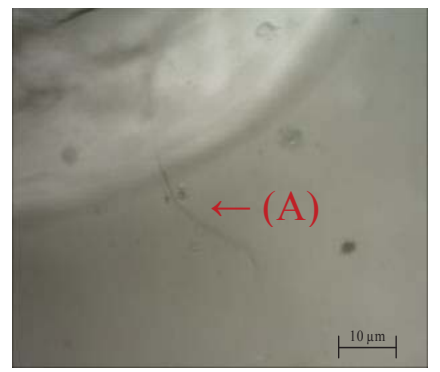

(a)

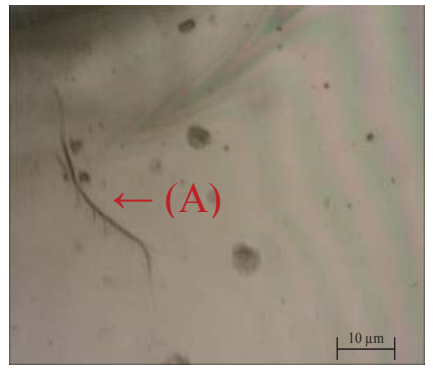

(b)
Figure 10. Morphology of the electrical tree growth on the cavity surface in the sample one after ageing time; (a) at 22 hours and (b) at 33 hours.

\section{B. Morphology on the different regions of the cavity surface}

After a period of 38 hours, the inner cavity was observed to darken with further increases in the surface roughness as shown in Figure 11. This result was most likely due to the influence of PD activity within the cavity, ultimately leading to electrical breakdown of the sample [8-10].

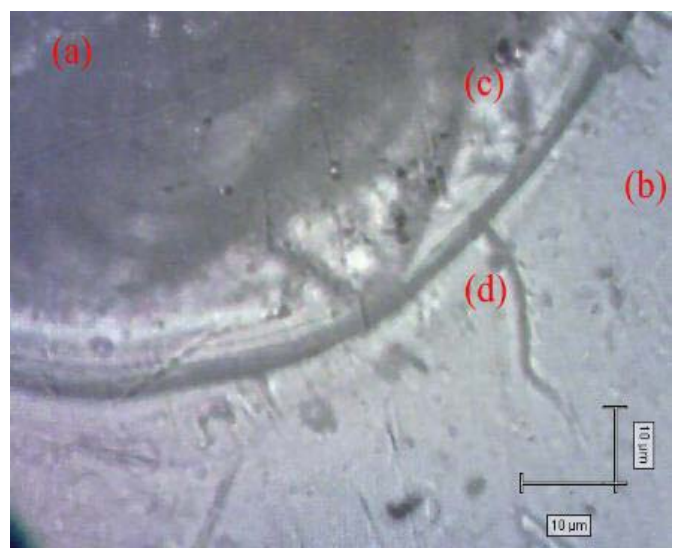

Figure 11. Morphology on the cavity surface in the sample one after ageing time at 38 hours; (a) an inner cavity, (b) an outside cavity surface, (c) a cavity surface and (d) an electrical tree channel. 


\section{Raman spectrums analysis}

The comparison of Raman spectrums on different regions of the cavity surface was investigated after 38 hours of ageing, correlating with the locations as shown in Figure 11. The spectra were obtained a minimal amount of fluorescence in the regions at the inner cavity and the outside cavity surface, whereas a large level of fluorescence at the cavity surface and the electrical tree channel were acquired as shown in Figure 12 (a), (b), (c) and (d) respectively. The appearance of fluorescence has been reported in previous work [11-13].

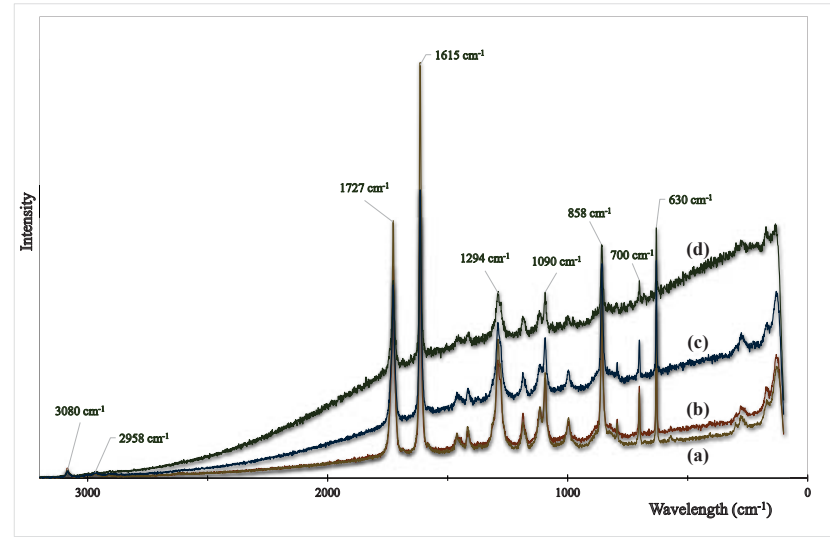

Figure 12. Comparison of Raman spectra on the cavity surface in the sample one after ageing time at 38 hours; (a) an inner cavity, (b) an outside cavity surface, (c) a cavity surface (d) an electrical tree channel.

\section{Raman spectrums on the cavity surface with ageing time}

The notable Raman spectrums on the cavity surface during ageing were obtained for the range of wavenumbers in the regions of $600 \mathrm{~cm}^{-1}$ to $1750 \mathrm{~cm}^{-1}$ and $2900 \mathrm{~cm}^{-1}$ to $3100 \mathrm{~cm}^{-1}$, including an unaged sample to provide a reference spectrum as shown in Figure 13 (a). The amount of fluorescence gradually increased with time period of ageing, the patterns are for the following ageing times: 22 hours, 33 hours and 38 hours as shown in Figure 13 (b), (c) and (d) respectively. This obtained result indicates that the ascending fluorescence associated with the surface roughness growth on the cavity as a result of the expanded period of ageing.

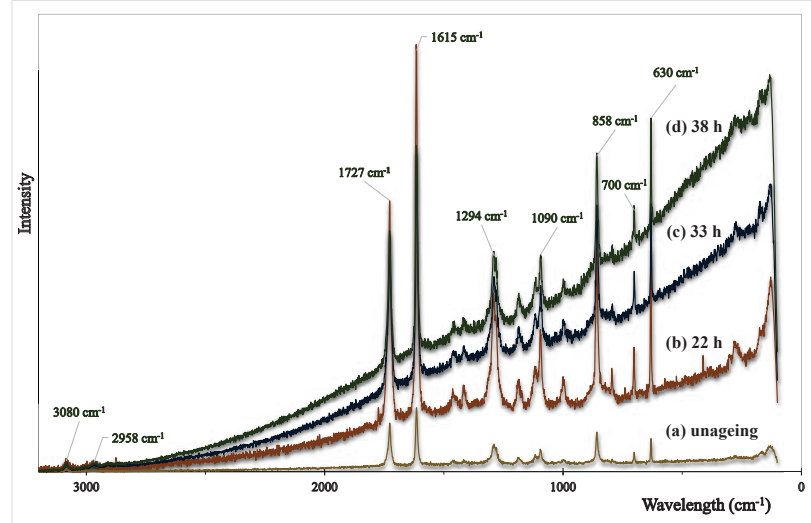

Figure 13. Comparison of Raman spectra on the cavity surface in the sample one after ageing time; (a) unageing sample, (b) ageing sample at 22 hours, (c) and (d) ageing sample at 33 hours and 38 hours respectively.

\section{CONCLUSIONS}

Analysis of experiment results for the sample one showed a transition of typical wing-like PRPD patterns from a cavity discharge to electrical tree growth and the attributed variation due to PD activity. Clear evidence of the SPMD mechanism was identified as the transition point, where the discharge magnitude was observed to rapidly reduce to less than $1 \mathrm{pC}$ in comparison with a previous magnitude of $115 \mathrm{pC}$.

This experiment indicated that the initial transition of PRPD patterns were noticed as the rabbit-like patterns and following turned into the turtle-like patterns for the sample two without electrical tree propagation on the cavity surface during ageing processes. When typical wing-like patterns and the SPMD mechanism were detected in the sample one. This appears to be due to the electrical tree growth. The Raman spectrums confirmed an increased level of fluorescence at the cavity surface and the electrical tree channel in relation to the elapsed time of the electrical ageing period indicating changes in the cavity surface structure.

\section{REFERENCES}

[1] P. H. F. Morshuis, "Degradation of solid dielectrics due to internal partial discharge: some thoughts on progress made and where to go now," IEEE Transactions on Dielectrics and Electrical Insulation, Vol. 12, pp. 905913, 2005.

[2] J. A. Hunter, P. L. Lewin, L. Hao, C. Walton, and M. Michel, "Autonomous classification of PD sources within three-phase $11 \mathrm{kV}$ PILC cables," IEEE Transactions on Dielectrics and Electrical Insulation, Vol. 20, pp. 2117-2124, 2013.

[3] F. Gutfleisch and L. Niemeyer, "Measurement and simulation of PD in epoxy voids," IEEE Transactions on Dielectrics and Electrical Insulation, Vol. 2, pp. 729-743, 1995.

[4] L. Wang, A. Cavallini, and G. C. Montanari, "Evolution of pd patterns in polyethylene insulation cavities under AC voltage," IEEE Transactions on Dielectrics and Electrical Insulation, Vol. 19, pp. 533-542, 2012.

[5] C. S. Kim, T. Kondo, and T. Mizutani, "Change in PD pattern with aging," IEEE Transactions on Dielectrics and Electrical Insulation, Vol. 11, pp. 13-18, 2004.

[6] Suwarno, "A comparison between void and electrical treeing discharges in polyethylene," Proceedings of the 6th International Conference on Properties and Applications of Dielectric Materials, pp. 493-496, 2000.

[7] K. Wu, Y. Suzuoki, T. Mizutani, and H. Xie, "Model for partial discharges associated with treeing breakdown: I. PDs in tree channels," Journal of Physics D: Applied Physics, Vol. 33, pp. 1197-1201, 2000.

[8] T. Tanaka, "Internal partial discharge and material degradation." IEEE Transactions on Electrical Insulation," Vol. EI-21, pp. 899-905, 1986.

[9] T. Ishida, Y. Mizuno, M. Nagao, and M. Kosaki, "Development and application of partial discharge analyzing system for swarming pulsive microdischarges," Proceedings of 3rd International Conference on Properties and Applications of Dielectric Materials (ICPADM), Vol. 2, pp. 676-679, 1991.

[10] K. Temmen, "Evaluation of surface changes in flat cavities due to ageing by means of phase-angle resolved partial discharge measurement," Journal of Physics D: Applied Physics, Vol. 33, pp. 603-608, 2000.

[11] A. S. Vaughan, S. J. Dodd, and S. J. Sutton, "A Raman microprobe study of electrical treeing in polyethylene," Journal of materials science, Vol. 39, pp. 181-191, 2004.

[12] N. A. Freebody, A. S. Vaughan, and P. L. Lewin, "Raman microprobe analysis and ageing in dielectrics," Journal of Physics: Conference Series, Vol. 183, pp. 1-5, 2009.

[13] N. A. Freebody, I. L. Hosier, and A. S. Vaughan, "Raman microprobe analysis of electrical treeing in silicone rubber," Proceedings of 17th International Symposium on High Voltage Engineering (ISH), Hannover, Germany, August 2011 [CD-ROM] 